Article

\title{
Two-Dimensional Ship Velocity Estimation Based on KOMPSAT-5 Synthetic Aperture Radar Data
}

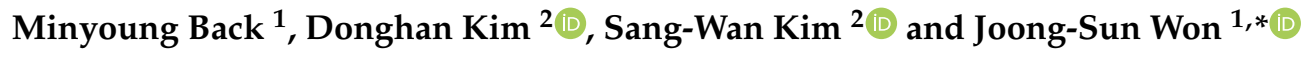 \\ 1 Department of Earth System Sciences, Yonsei University, Seoul 03722, Korea; min0100@yonsei.ac.kr \\ 2 Department of Geoinformation Engineering, Sejong University, Seoul 05006, Korea; \\ legokdh@naver.com (D.K.); swkim@sejong.edu (S.-W.K.) \\ * Correspondence: jswon@yonsei.ac.kr
}

Received: 13 May 2019; Accepted: 19 June 2019; Published: 21 June 2019

\begin{abstract}
Continuously accumulating information on vessels and their activities in coastal areas of interest is important for maintaining sustainable fisheries resources and coastal ecosystems. The speed, heading, sizes, and activities of vessels in certain seasons and at certain times of day are useful information for sustainable coastal management. This paper presents a two-dimensional vessel velocity estimation method using the KOMPSAT-5 (K5) X-band synthetic aperture radar (SAR) system and Doppler parameter estimation. The estimation accuracy was evaluated by two field campaigns in 2017 and 2018. The minimum size of the vessel and signal-to-clutter ratio (SCR) for optimum estimation were determined to be $20 \mathrm{~m}$ and $7.7 \mathrm{~dB}$, respectively. The squared correlation coefficient $R^{2}$ for vessel speed and heading angle were 0.89 and 0.97 , respectively, and the root-mean-square errors of the speed and heading were $1.09 \mathrm{~m} / \mathrm{s}$ ( $2.1 \mathrm{knots})$ and $17.9^{\circ}$, respectively, based on 19 vessels that satisfied the criteria of minimum size of vessel and SCR. Because the K5 SAR is capable of observing a selected coastal region every day by utilizing various modes, it is feasible to accumulate a large quantity of vessel data for coastal sea for eventual use in building a coastal traffic model.
\end{abstract}

Keywords: coastal traffic monitoring; SAR; two-dimensional ship velocity; Doppler parameters; KOMPSAT-5

\section{Introduction}

It is necessary to accumulate data on ships and boats within coastal areas of interest for integrated coastal and marine resource management. Conservation and sustainable resource use are important issues in coastal areas. One of the main concerns of many Asian countries is maintaining sustainable fishery resources in their coastal oceans. For monitoring ships and boats, it has been strongly recommended that vessels be equipped with an automatic identification system (AIS). Most large ships and fishing vessels can be identified and monitored through the AIS. However, the areal coverage of the AIS is limited to only approximately $40 \mathrm{~km}$ from a ground-based receiver. More importantly, many relatively small vessels, or some illegal ones, either simply do not have an AIS or intentionally switch off the system to avoid exposing their location and activities. Such uncontrolled fishing activities at coastal fisheries not only deplete fishery resources but also seriously damage the coastal ecosystem. Therefore, for managing marine resources, governments are building a database of vessel information such as the total number, types, locations, and activities in coastal areas of interest according to fishing calendars of major species and time of day.

Detecting and retrieving the velocity of ground moving targets is an important application of synthetic aperture radar (SAR). Detecting vessels from space using an SAR system has been proved particularly effective for ships larger than $30 \mathrm{~m}$ [1,2]. However, monitoring and building a seasonal database of coastal vessels requires not only detecting and locating vessels, but also collecting data 
including their heading, velocity, and type. In addition to performing the detection itself, it is essential to estimate the two-dimensional velocity components (both range and azimuth) from which the heading and velocity data for a ship is obtained. Many previous works such as $[1,3,4]$ proposed various methods by which to detect ships and estimate range (or radial) velocity; the details of such methods are discussed in Section 2.2.1. Various methods have also been proposed for estimating the azimuth (along-track) velocity of ground moving targets in association with Doppler rate estimations. While many researchers have focused on detecting vessels in coastal regions, only a few such as [5] reported and discussed retrieving the two-dimensional velocity of vessels at sea. Furthermore, the accuracy of two-dimensional vessel velocity derived using spaceborne SAR systems has rarely been discussed. This paper presents an integrated method for retrieving the two-dimensional velocity of vessels and evaluates the estimation accuracy based on speed and heading. Limitations of the method are discussed in terms of the minimum size of the vessels, minimum speed, and signal-to-clutter ratio (SCR). A high-resolution spaceborne SAR system is suitable here because fishing vessels are generally small; therefore, the KOMPSAT-5 (K5) X-band SAR was used in this study. Field campaigns were carried out in 2017 and 2018 to obtain SAR data images of vessels; their headings and speeds were controlled using recorded global positioning system (GPS) tracks. Other vessels with an AIS within the images were also utilized; 32 vessels were used to evaluate accuracy. Because the raw signal data acquired by most current high-resolution SAR systems are simply not available to public users, this study presumes that only SAR single-look complex (SLC) data, not raw signals, are available.

It is well known that a moving target in an SAR SLC image includes three features: Azimuth shift, azimuth defocus, and range smear [6]. While these features distort SAR images, they have been exploited as ground moving targets indicators (GMTIs) and to retrieve the velocity of a target [7]. The velocity of vessels can be measured from SAR signals by measuring the residual Doppler frequency components directly $[2,8]$. The characteristics of the targets and clutter in these residual Doppler frequency estimations of vessels at sea would be very different from those of vehicles on land in terms of the following: (1) The size of the target; a ship is usually larger than an object on land; (2) the SCR at sea, which is generally larger than that on land, although the latter depends greatly on the state of the sea and system parameters including frequency and polarization; (3) the wake produced by the movement of a ship or boat across the surface of water, which helps to detect it and determine its heading [3]. These three features make it easier to detect and estimate the residual Doppler frequency of moving targets at sea. However, there are some negative features to consider, including the following: (1) The forward speed of a ship, which is generally lower than that of land vehicles, implies that the residual Doppler frequency associated with the target motion is relatively small and difficult to discriminate from the clutter bandwidth; and (2) the roll and pitch motions of a ship, which make scattering centers move continuously during the SAR observation [8].

The organization of this paper is as follows. In Section 2, the method, SAR data, and field campaigns are described briefly. The application results are reported in Section 3 and the discussion and conclusions follow in Sections 4 and 5, respectively.

\section{Method and Data}

\subsection{SAR and Field Data}

For the SAR and sea-truth data acquisition, field campaigns were carried out twice, i.e., in 2017 and 2018. Figure 1 shows the K5 SLC image acquired at 06:17 local time on 26 September 2017. Table 1 lists the K5 SAR system specifications, which are required for the Doppler parameter estimation. A follow-up campaign was carried out at 05:47 local time on 4 October 2018. September and October are the high season months for crab fishing. The sea state on both days of $\mathrm{K} 5$ data acquisition was calm. Table 2 summarizes the sea state on the days of data acquisition. The wind speed was $2.0 \mathrm{~m} / \mathrm{s}$ with a wave height of $0.2 \mathrm{~m}$ on the $2017 \mathrm{~K} 5$ data acquisition, and it was $1.7 \mathrm{~m} / \mathrm{s}$ with a wave height of $0.3 \mathrm{~m}$ on the 2018 campaign. During the 2017 K5 SAR observation, two vessels, whose headings and 
speeds were precisely controlled by the GPS and AIS recordings, were deployed within the scene area. The AIS data were used to measure the positions and velocities for the rest of the vessels.

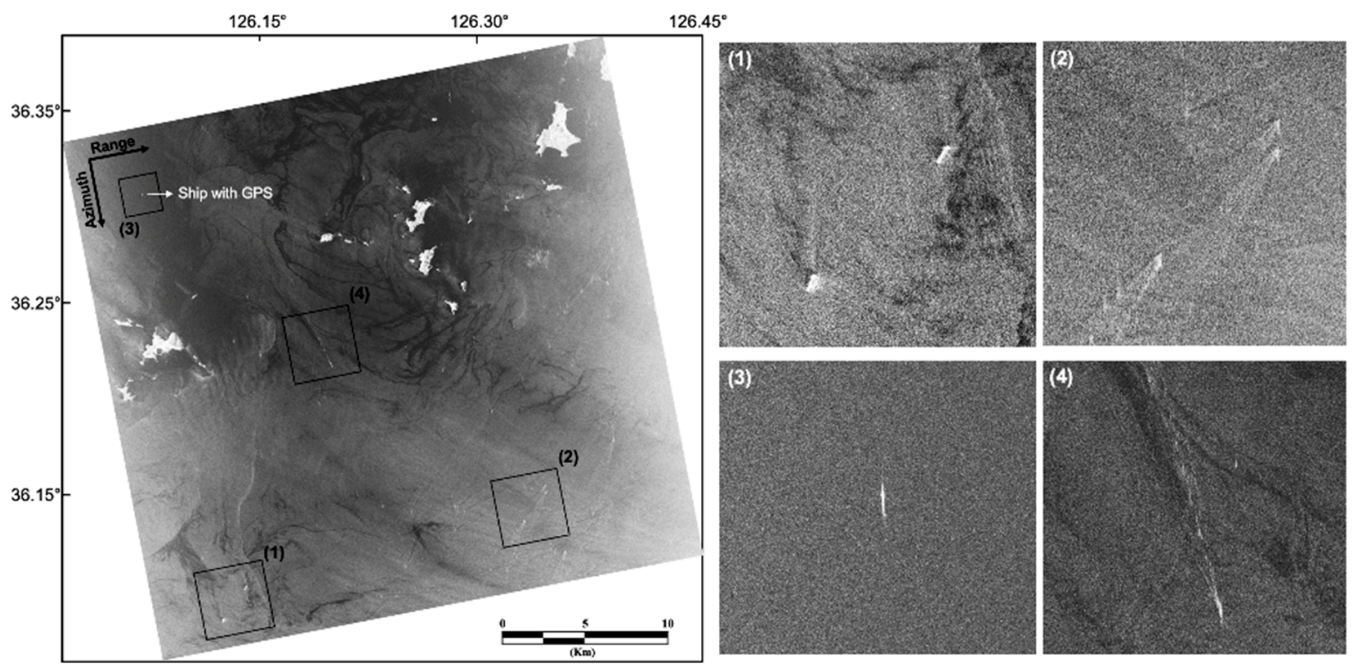

(a)

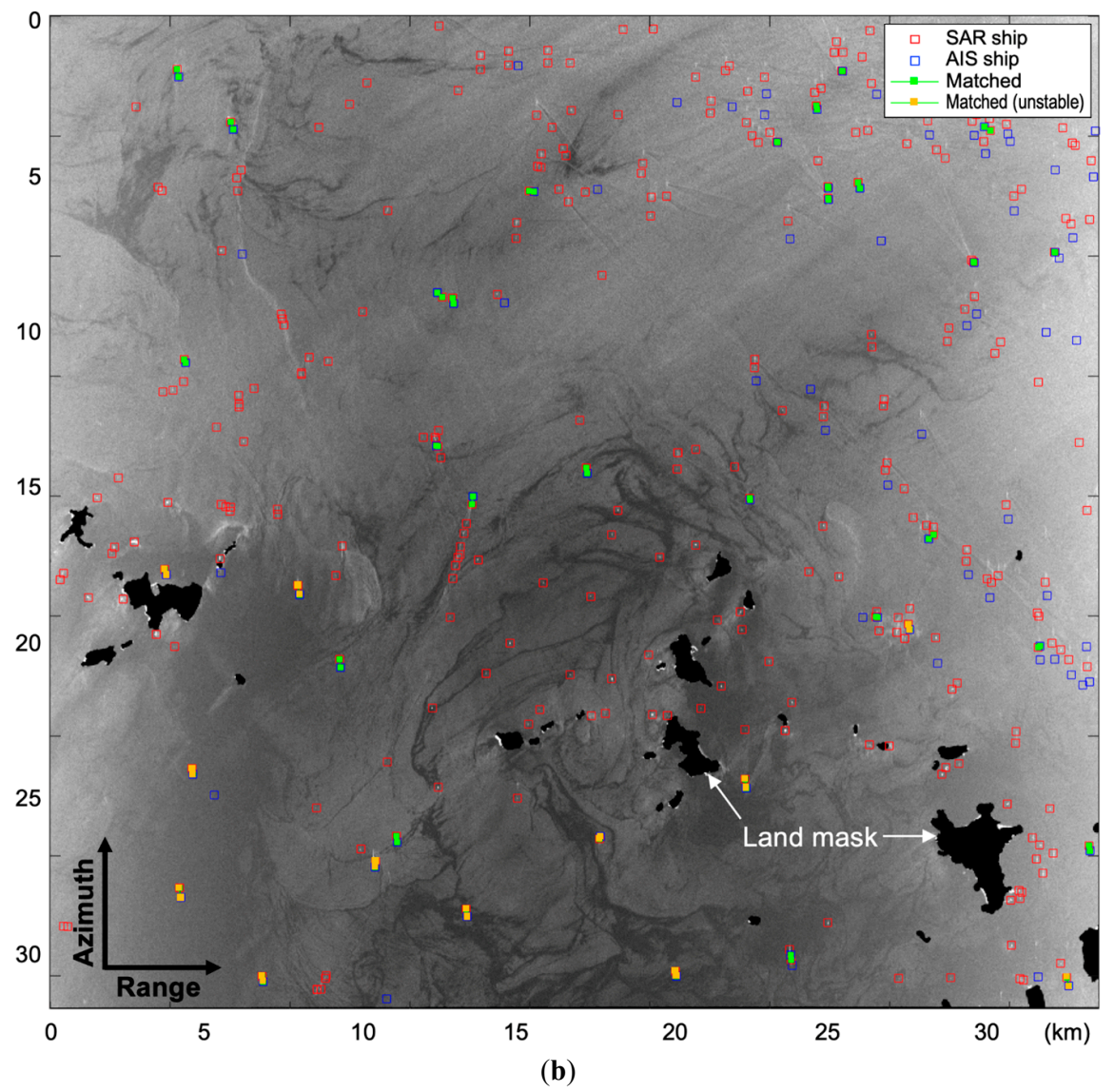

Figure 1. (a) KOMPSAT-5 (K5) synthetic aperture radar (SAR) image acquired on 26 September 2017 in which vessels and ship wake patterns as well as sea surface features are well rendered. (b) Vessels detected by K5 SAR (red squares) and traced by the automatic identification system (AIS) (blue squares). The squares filled with green represents vessels that are commonly detected by both the SAR and AIS and used for velocity evaluation. The squares filled with orange presents vessels commonly detected by both the SAR and AIS but cannot not be used for velocity estimation because the AIS signals were too unstable to restore velocities and headings. 
Table 1. Summary of KOMPSAT-5 SAR system specifications.

\begin{tabular}{ccc}
\hline Parameters & Values & Symbols \\
\hline Polarization & $\mathrm{HH}$ & \\
Wavelength & $0.0310 \mathrm{~m}$ & $\lambda$ \\
Nominal altitude & $550 \mathrm{~km}$ & $H$ \\
Antenna velocity & $7664.2 \mathrm{~m} / \mathrm{s}$ & $V_{s}$ \\
Pulse repetition frequency & $3787.9 \mathrm{~Hz}$ & $P R F$ \\
Azimuth integration time & $0.5847 \mathrm{~s}$ & $T_{\tau}$ \\
Azimuth bandwidth & $3100 \mathrm{~Hz}$ & $B_{a}$ \\
Azimuth resolution & $2.19 \mathrm{~m}$ & $\Delta x$ \\
Azimuth pixel spacing & $1.8509 \mathrm{~m}$ & $d x$ \\
Range $^{(1)}$ resolution & $2.14 \mathrm{~m}$ & $\Delta y$ \\
Range $^{(1)}$ pixel spacing & $1.0519 \mathrm{~m}$ & $d y$ \\
\hline${ }^{(1)}$ The "Range" in Table 1 refers to the "Slant range.".
\end{tabular}

Table 2. Summary of sea states measured from oceanographic buoy and automatic weather station.

\begin{tabular}{ccc}
\hline Date & Parameters & Values \\
\hline 26-09-2017 & Wind direction & $101.4^{\circ}$ \\
06:17 (local time) & Wind speed & $2.0 \mathrm{~m} / \mathrm{s}$ \\
& Wave height & $0.2 \mathrm{~m}$ \\
\hline \multirow{2}{*}{ 04-10-2018 } & Wind direction & $9.9^{\circ}$ \\
05:45 (local time) & Wind speed & $1.7 \mathrm{~m} / \mathrm{s}$ \\
& Wave height & $0.3 \mathrm{~m}$ \\
\hline
\end{tabular}

As shown in Figure 1, the K5 X-band SAR with a nominal ground resolution of $3 \mathrm{~m}$ provides an excellent rendition of vessels and ship wake patterns. From the K5 SAR data, vessels were detected by adopting a sub-aperture mismatching method [1,9] with a combination of human visual attention system and constant false alarm rate, which was developed for operational ship surveillance using the K5 SAR. Concerning the AIS data, many vessels simply did not have or switched off the AIS, and the AIS data of some vessels provided only sporadic positional data from which it was almost impossible to trace exact locations and velocities. The matching of the K5-detected ships with the AIS data was performed using the coherent point drift algorithm [10]. The vessels traced by the AIS were heavily outnumbered by those imaged by the SAR, as summarized in Table 3. While 347 vessels were detected from the two K5 SAR scenes, only 117 vessels were traceable by the AIS. Among the SAR-detected vessels, some false-alarms, for instance in the left side of the scene center, were included so that the true number of SAR detected vessels would be fewer than 347. The K5 SAR also did not detect a total of 69 vessels whose sizes were generally smaller than a few tens of meters. However, the precise estimation of SAR-based detection rate is beyond the scope of this study, and thus, no further refinement steps were applied. Out of 416 vessels within the two scenes, the AIS provided information on only 117 vessels; this is approximately $28 \%$ of all vessels. These results clearly demonstrate that using the AIS alone is not enough for complete monitoring of vessels and collecting their information in this coastal area where many vessels are either unable to provide stable AIS signals or involved in illegal fishing activities.

Table 3. Summary of vessels detected by K5 SAR and traced by AIS data.

\begin{tabular}{ccccccc}
\hline Date & $\begin{array}{c}\text { K5 Scene } \\
\text { No. }\end{array}$ & $\begin{array}{c}\text { Detected by } \\
\text { K5 SAR }\end{array}$ & $\begin{array}{c}\text { Traced } \\
\text { by AIS }\end{array}$ & $\begin{array}{c}\text { Common to K5 } \\
\text { SAR and AIS }\end{array}$ & $\begin{array}{c}\text { Not Detected } \\
\text { by K5 SAR }\end{array}$ & $\begin{array}{c}\text { Not Detectedby } \\
\text { AIS }\end{array}$ \\
\hline $25-09-2017$ & 000010 & $287^{(2)}$ & $99^{(1)}$ & $38^{(1)}$ & 61 & $249^{(1)}$ \\
$03-10-2018$ & 004042 & $60^{(2)}$ & $18^{(1)}$ & 10 & 40 & 69 \\
\hline \multicolumn{2}{c}{ Total (416) } & $347^{(2)}$ & $117^{(1)}$ & $48^{(1)}$ & $299^{(1)}$ \\
\hline
\end{tabular}

(1) It includes AIS detected 12 vessels, but their signals were too unstable to restore velocities and headings. ${ }^{(2)}$ The total number of K5 SAR detected vessels includes false alarms mainly around ship wake. 
Among the 416 vessels, 48 were observed by both the AIS and K5 SAR during the two field campaigns. Among the total of 48 commonly detected vessels, the AIS signals from 12 vessels were too unstable to restore vessel's velocities and headings. Because vessels in motion at the moment when the K5 SAR data are acquired are useful for this study, 26 vessels with observable speeds were finally selected and used for the two-dimensional velocity estimation and accuracy evaluation.

\subsection{Method}

Estimating both the range and azimuth velocity of a vehicle is necessary to obtain information on its speed and heading. It is well known that the range velocity of a moving object contributes to its Doppler frequency, which results in an azimuth shift of the ship in an SAR image. The azimuth component of a moving ship contributes to image blurring by chirp rate mismatching; therefore, it is necessary to estimate the residual Doppler rate.

\subsubsection{Point Target Spectrum in Two-Dimensional Frequency Domain}

To estimate the Doppler parameter, it is important to understand the point target spectrum in the two-dimensional frequency domain. A point target in the SLC data is represented by the range and azimuth compressed signals. A range and azimuth compressed point moving target in the SAR SLC data can be described by a frequency spectrum $S_{M T}\left(f, f_{\tau}\right)$ in a two-dimensional frequency domain as follows [11]:

$$
\begin{aligned}
S_{M T}\left(f, f_{\tau}\right) \simeq & \operatorname{rect}\left(\frac{f}{B_{r}}\right) \operatorname{rect}\left(\frac{1}{B_{a}} \cdot\left[f_{\tau}-\alpha \cdot\left(1+\frac{f}{f_{0}}\right)\right]\right) \\
& \times \exp \left\{-i 2 \pi \Phi_{\text {res }}\left(f, f_{\tau}\right)\right\}
\end{aligned}
$$

where $f$ and $f_{\tau}$ are the range frequency and Doppler (or azimuth) frequency, respectively; $f_{0}$ is the carrier frequency; and $B_{r}$ and $B_{a}$ are the range bandwidth and Doppler bandwidth, respectively. For a target fixed on the ground, the residual phase $\Phi_{r e s}\left(f, f_{\tau}\right)$ and $\alpha$ in Equation (1) must be zero if an ideal focusing is done. For a ground moving target, the parameter $\alpha$ is given by

$$
\alpha=-2 \frac{v_{r}}{\lambda} \sin \theta
$$

where $v_{r}$ is the target's ground range velocity, $\lambda$ is the wavelength, and $\theta$ is the antenna elevation angle. The Doppler centroid is a mean Doppler frequency over range bins, which causes the Doppler-shift of a target positioned in the antenna boresight direction [12]. The parameter $\alpha$ contributes to the Doppler centroid as an additional Doppler frequency due to target motion as in Equation (4). The residual phase $\Phi_{\text {res }}\left(f, f_{\tau}\right)$ in the two-dimensional frequency domain can be approximated further from the original equations described by [13] up to the second order of Doppler frequency as follows:

$$
\Phi_{r e s}\left(f, f_{\tau}\right) \approx \Phi_{1}\left(f, f_{\tau}\right)+\Phi_{2}\left(f, f_{\tau}\right)+\Phi_{3}(f)
$$

where

$$
\begin{gathered}
\Phi_{1}\left(f, f_{\tau}\right) \approx+\frac{(1+\beta)}{K_{a}} \cdot \alpha \cdot\left[f_{\tau}-\alpha \cdot\left(1+\frac{f}{f_{0}}\right)\right] \\
\Phi_{2}\left(f, f_{\tau}\right) \approx-\frac{\beta}{2 K_{a}} \cdot f_{\tau}^{2} \cdot\left[1-\left(\frac{f}{f_{0}}\right)+\left(\frac{f}{f_{0}}\right)^{2}\right] \\
\Phi_{3}(f) \approx+\frac{(1+\beta)}{2 K_{a}} \cdot \alpha^{2} \cdot\left[1+\left(\frac{f}{f_{0}}\right)\right]
\end{gathered}
$$

where the Doppler rate of the stationary target $K_{a}$ is approximated as

$$
\frac{1}{K_{a}} \simeq \frac{\lambda R_{0}}{2 V_{s}^{2}}
$$


at a distance of $R_{0}$ from the antenna, which moves with a velocity of $V_{S}$ along the azimuth direction. The azimuth velocity parameter $\beta$ is given by

$$
\beta=\left(2 \frac{v_{a}}{V_{s}}-\frac{a_{r}}{V_{s}^{2}} R_{0} \sin \theta\right)
$$

where $v_{a}$ is the target's ground azimuth velocity, and $a_{r}$ is the range component of acceleration. Thus, the two-dimensional velocity retrieval from the SLC data is summarized as an estimation of $\alpha$ and $\beta$ from Equations (4) and (5), respectively. It is thoroughly understood that the $\alpha$ term is involved in the Doppler frequency shift in Equation (4) and results in an azimuth shift of the target in the focused SAR image. The $\beta$ term contributes to the residual Doppler rate as indicated in Equation (5), which results in an azimuth defocus. It must be noted that the $\beta$ term with a squared Doppler frequency $f_{\tau}{ }^{2}$ in (5) is coupled with a first- and second-order of range-frequency terms $f$ and $f^{2}$. More details on the properties of each term will be discussed in the following subsections.

\subsubsection{Range Velocity Estimation}

As in Equations (2) and (4), the range velocity component of a moving vessel can be measured using Doppler centroid estimation. Various methods have been developed for this; conventional methods including energy balancing [14], the average cross-correlation coefficient method [12], the spectral fit approach [15], multi-look cross-correlation, and the multi-look beat frequency algorithm [16] were developed mainly for SAR focusing. The performance of these algorithms was discussed in detail by [12]. More recently, a Radon transform-based Doppler parameter estimator was proposed by [17]. A method that examined the skew of the received signal in the two-dimensional frequency domain was discussed to resolve the unaliased range velocity component [18]. Although these methods provide very reliable estimates, they were basically developed for SAR focusing and must be averaged over several range bins. To measure the Doppler frequency of vessels, the Doppler parameters must be calculated using less than 20 to 30 range bins. When a small boat moves parallel to the azimuth, the range bins suitable for estimation are reduced again, to less than 10. A joint time-frequency analysis (JTFA), which uses a mean conditional frequency from the Wigner-Ville distribution, is useful for a point-like target $[19,20]$. The statistical model for the echo backscattered by the homogeneous ground surface can be modelled as a Gaussian function in the Wigner-Ville distribution [20,21]. Based on this property, [13] proposed a Doppler spectrum tracing method in a joint time-frequency domain. They concluded that their method was very useful for fast-moving targets but only effective if the target speed was at least $1.4 \mathrm{~m} / \mathrm{s}$ (2.7 knots) or higher. In addition, JTFA-based methods normally require azimuth un-compressed signals. However, both range- and azimuth-compressed signals in the SLC are only available to normal users. Recently, [2] proposed a sophisticated Doppler centroid estimation algorithm for improving ship detection with radial velocity estimation; in this method, the characteristics of the Doppler spectrum of ships were discussed thoroughly. Their method basically exploited the clutter-lock algorithm and demonstrated an accurate retrieval of the radial velocity of ships with a root-mean-square deviation of less than $5 \%$ at ship speeds higher than or equal to $2 \mathrm{~m} / \mathrm{s}$ (3.9 knots).

There is another approach for estimating the velocity of vessels on the ocean; this exploits ships' wakes. When a moving vessel generates a wake on the sea, its radial motion (or range velocity component) causes an azimuth shift and makes the wake in the SAR imagery off the vertex. If the vertex of a ship's wake is clearly imaged, the azimuth shift can be computed to measure the ship's radial velocity [22]. The Kelvin wave pattern, which is the most external wake component characterized by transverse, divergent, and cusp waves, is particularly useful for the SAR-based vessel radial velocity estimation [3]. The details of wake component detection and vessel velocity estimation were discussed by $[3,4,23]$. Although the ship-wave-based methods are very effective for detecting and estimating the radial velocity of large and fast-moving vessels with a clear wake pattern, they are 
usually ineffective for small and slow-moving vessels. In addition, the method is limited to the radial velocity component only.

Thus, the most robust and precise approach for the range velocity estimation of a moving vessel would be a direct estimation of the residual Doppler frequency given by Equations (1), (2), and (4). However, it is often difficult to estimate the central frequency in the Doppler frequency domain directly, mainly because of noise [8]. This study uses the method proposed by [8]; where further details of the method used can be found. The method adopted is to estimate $\alpha$ defined by the Equation (2) via linear least squares instead of direct estimation of residual Doppler frequency, which provides optimum estimates from sets of azimuth subsamples that have different azimuth temporal distances [8]. The accuracy of estimating residual Doppler frequencies depends on factors including the SCR and the speed, size, and roll and pitch the target ship. The method adopted is suitable for measuring the residual Doppler frequency of moving ships at a high accuracy [8].

\subsubsection{Azimuth Velocity Estimation}

Compared to the many methods proposed for range velocity estimation, few have been developed to estimate residual Doppler rate, particularly from SAR SLC data, for moving vessels. Most methods exploit raw or range-compressed signals. One conventional approach is based upon the image-blurring effect caused by the along-track velocity component. A Doppler rate detector was proposed and its performance compared with that of a two-channel method [24]. Another conventional approach is based on the maximum likelihood estimation of the parameters of quadratic frequency modulated signals $[25,26]$. However, those methods have disadvantages including computational inefficiency and ineffectiveness for noisy signals in which the local maxima make it difficult to find a global maximum. The computational efficiency and performance were significantly improved by introducing a cubic phase function (CPF) [27]. Since the first introduction of the CPF, many improved methods have been developed to improve efficiency and to estimate higher-order phase polynomials [28,29]. Although those CPF-based methods are computationally very competitive in terms of a high performance for azimuth uncompressed signals, they are not feasible for Doppler rate estimation of vessels at sea. This is because the residual Doppler rate of moving vessels presented by the azimuth compressed SAR SLC signals are limited to a few tens of azimuth samples. Performance improves slightly when the CPF method is applied in the Doppler domain compared to the slow-time domain. Of the various approaches, JTFA has been popularly applied to measure the motion of an object directly from a chirp signal and has been successful at retrieving velocity by estimating the Doppler frequency rate of a moving object in the time-frequency domain [21,30]. The JTFA demonstrated the potential for extracting the time sequence of motion parameters [31,32]. It is possible to measure the velocity of ground-moving vehicles with an error of less than $5 \%$ for velocities higher than $3 \mathrm{~m} / \mathrm{s}$ [13].

SLC data of high-resolution SAR systems are only available to public users. One conventional approach applicable to single-channel SLC data is the multi-look misregistration method proposed by [9]. This sub-aperture approach is very effective for the detection of moving vessels. An improved method was proposed for a bistatic SAR system using TerraSAR-X and TanDEM-X and tested for moving ship velocity estimation with an accuracy of $2.23 \mathrm{~m} / \mathrm{s}$ [5]. This approach is very effective with a superior accuracy for sufficiently large vessels, but not for small ones under low-SCR conditions. Most of all, it is a multi-channel approach for providing an improved accuracy, but such multi-channel is not popular for most operational spaceborne SAR systems. Instead of the conventional approach, a method based upon the fractional Fourier transform (FrFT) and minimum entropy was adopted in this study. The FrFT is a generalization of the Fourier transform, and it is defined as [33]

$$
S_{a}(u)=\frac{\exp \left(j \frac{\theta}{2}\right)}{\sqrt{j \sin \theta}} \exp \left(-\frac{j}{2} u^{2} \cot \theta\right) \int_{-\infty}^{\infty} \exp \left(-\frac{j}{2} x^{2} \cot \theta-\frac{j u x}{\sin \theta}\right) s(x) d x
$$


where the rotation angle $\alpha$ is a function of the fractional transformation order $a$ given by

$$
\theta=\frac{\pi}{2} a
$$

When $\theta=\frac{\pi}{2}$, i.e., $a=1$, the FrFT becomes the ordinary Fourier transform while the transform kernel reduces to an identity operation when $\theta=0$. Because the transform kernel in Equation (9) is a chirp, the FrFT is particularly useful for applications involving chirp signals such as signal enhancement problems with accelerating sinusoidal sources where the Doppler effects generate chirp signals and a frequency shift. The FrFT can process chirp signals better than the ordinary Fourier transform [34,35]. This is because a chirp signal forms a line in the time-frequency plane, and therefore, there exists an order of transformation in which such signals are compact. Chirp signals are not compact in the time or spatial domain. Thus, we can extract the signal easily in an optimum fractional Fourier domain [34]. The FrFT is an effective and efficient method for estimating a moving target parameter, particularly the residual Doppler rate via residual azimuth compression [35-37]. Residual focusing via FrFT is particularly effective for an isolated point target but probably not for a group of targets with different chirp rates. Thus, the FrFT is suitable for the residual focusing of an isolated moving vehicle or vessel.

The application of the FrFT to SAR SLC data requires a criterion for assessing the degree of azimuth compression. The minimum entropy is a very useful parameter for determining the optimal azimuth focusing [38,39]. A combination of FrFT and minimum-entropy criteria would be a powerful tool when combined. Radar images suffer image focus degradation in the presence of phase errors in the received signal due to the unknown platform motion or target motion. An optimal focusing can be achieved by applying the entropy minimization principle, which is effective for estimating phase errors under conditions of low signal-to-noise ratio, low image contrast, and substantial phase errors $[38,40]$. Let us consider the signal of an $(m, n)$ th pixel in the range- and azimuth-compressed SLC SAR image given by

$$
s_{m, n}(\tau ; \varphi)=\frac{1}{N} \sum_{k=0}^{N-1} S_{m, k}\left(f_{\tau}\right) \exp \left\{j \varphi_{k}\right\} \exp \left\{j 2 \pi \frac{k n}{N}\right\}
$$

where $N$ is the number of azimuth samples over the integration time, $S_{l}\left(f_{\tau}\right)$ the azimuth Fourier transform of the $l$ th scatterer, and $\varphi_{k}$ the phase error. The index $n$ corresponds to either the azimuth (or cross-range) or Doppler coordinate. Then, the global minimum-entropy criterion for the SAR SLC is defined by $[39,40]$

$$
\Phi(\varphi)=-\frac{1}{E_{s}} \sum_{m, n}\left|s_{m, n}\right|^{2} \ln \left|s_{m, n}\right|^{2}+\ln E_{s}
$$

where

$$
E_{s}=\sum_{m, n}\left|s_{m, n}\right|^{2}
$$

Then, the minimum-entropy phase estimate is defined as

$$
\hat{\varphi}=\underset{\varphi}{\operatorname{argmin}} \Phi(\varphi) .
$$

The residual Doppler rate $\beta$ defined by Equations (5) and (8) can be obtained from the phase estimate $\hat{\varphi}$ such as

$$
\frac{\beta}{K_{a}}=\frac{N}{P R F^{2} \tan \left(\frac{\pi}{2}(1-\hat{\varphi})\right)}
$$

where $N$ is the number of azimuth samples, $P R F$ the pulse-repetition-frequency or azimuth sampling frequency. The number of samples $N$ depends the size of a given target but generally less than or equal to 64 is recommended for an application to vessel. It would provide a powerful tool for residual 
Doppler rate estimation when the FrFT and minimum-entropy criteria are combined. In addition to this approach, it must be emphasized that it is mandatory to apply the method to an averaged azimuth line over neighboring range bins. The residual Doppler rate terms are given by Equations (1) and (5). From Equation (5), it is evident that the residual Doppler frequency rate $\beta$ in the Doppler frequency domain can only be properly estimated when the range frequency is zero. The zero-frequency component or DC component can be obtained simply by averaging the azimuth signals over the range bins.

\section{Application Results}

As mentioned in the previous section, many vessels were not traceable by the AIS alone. There are various reasons for this. One is that some signals from onboard AIS transponders are extremely unstable and/or irregular in terms of properly tracing the vessel they correspond to. Additionally, many vessels, particularly small fishing vessels, frequently switch off their onboard AIS because they do not intend to divulge their exact fishing sites. Some vessels involved in illegal activities also switch off their AISs. It was clear that the AIS alone was not enough for building statistical data on coastal vessels; additional information, such as SAR, would be a useful source of coastal traffic management data.

A total of 26 vessels detected by both the AIS and K5 SAR were used for accuracy evaluation. By applying the methods described in the previous section, the ground range (or radial) and azimuth (or along-track) velocity components of each vessel were estimated first. When the estimated Doppler frequency and Doppler frequency rate were converted into ground velocity components, the geometry of spacecraft orbit should be considered rather than a simple flat-Earth approximation [41]. From our experience, the flat-Earth approximation accounts for approximately $7 \%$ to $11 \%$ of the velocity errors retrieved. The K5-derived ground range and azimuth velocities versus AIS/GPS velocities are displayed in Figure 2a,b, respectively. The squared correlation coefficient $R^{2}$ between the AIS/GPS and K5-derived azimuth velocities was 0.94 . The root-mean-square error (RMSE) of the results was $1.33 \mathrm{~m} / \mathrm{s}$ with a slope of 1.04 . High-speed vessels faster than $8 \mathrm{~m} / \mathrm{s}$ generally contributed to the errors as observed in Figure $2 \mathrm{~b}$. When the acceleration in Equation (8) is not negligible, the estimation error of the azimuth velocity becomes large. Concerning the estimated range velocity, the squared correlation coefficient $R^{2}$ between the AIS/GPS and K5-derived velocities was 0.90. The RMSE of the results was $1.15 \mathrm{~m} / \mathrm{s}$ with a slope of 0.90 . A slope smaller than 1 implies that the $\mathrm{K} 5$-derived vessel range velocity component was slightly underestimated. Although the RMSE of the radial velocity component is smaller than that of the along-track component, a slope close to 1 is very important for future applications. The roll motion of the ship superstructure including the bridge would be one of the main error sources on the range velocity estimation. Potential error sources of the velocity estimation will be discussed later. The vessel speed and heading were estimated by the two velocity components. The RMSEs of the estimated vessel speed and heading were $1.52 \mathrm{~m} / \mathrm{s}$ and $24.3^{\circ}$, respectively.

The accuracy of vessel velocity estimation depends on various conditions including the SCR, speed of ship, size of ship, and roll and pitch of ship. Therefore, it is also important to determine favorable conditions for vessel velocity estimation of spaceborne high-resolution SAR systems. Figure 3 shows the accuracy of the SAR-derived velocity according to the size of vessel and SCR. It was reported that size is very important for detecting vessels and the detection rate is improved for vessels larger than $30 \mathrm{~m}$ [1]. Similar to the ship detection case described by [1], the velocity estimation error also decreases as the size of vessel increases as shown in Figure 3a. To determine the minimum size of vessel, the relationship between the size of vessel and the estimation was examined. The relation can be represented by an exponential fitted function as shown in Figure 3a. The crossing point of the fitted line with the RMSE of speed $1.52 \mathrm{~m} / \mathrm{s}$ is indicated as $19.9 \mathrm{~m}$. Thus, a vessel size larger than $20 \mathrm{~m}$ is recommended for the estimation of velocity. The relationship between the velocity estimation error and SCR was similarly obtained. The measurement accuracy is improved as the SCR increases (i.e., strong signals from a given vessel while low backscattering from the surrounding sea surface). As shown in Figure 3b, an SCR greater than $7.7 \mathrm{~dB}$ is the required condition for the velocity estimation. 
These two conditions can be considered for ship velocity estimation from spaceborne X-band SAR systems in coastal areas.

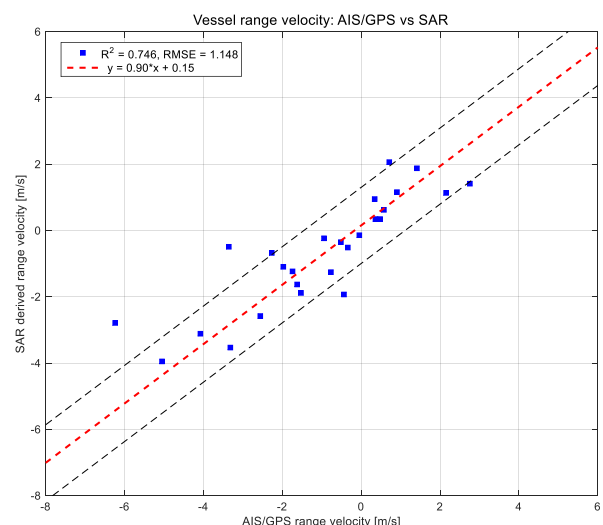

(a)

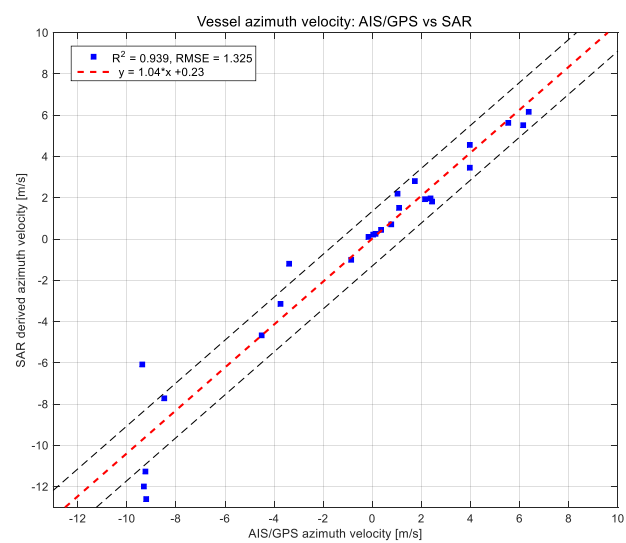

(b)

Figure 2. K5 SAR-derived vessel velocities versus AIS/global positioning system (GPS) velocities: (a) ground range velocity and (b) azimuth velocity. The squared correlation coefficient R2 are 0.75 and 0.94 for the range and azimuth velocities with root-mean square errors (RMSE) of $1.15 \mathrm{~m} / \mathrm{s}$ and $1.33 \mathrm{~m} / \mathrm{s}$, respectively.

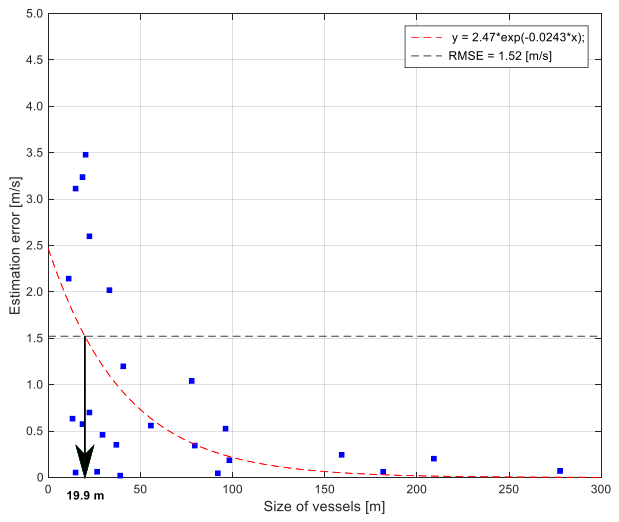

(a)

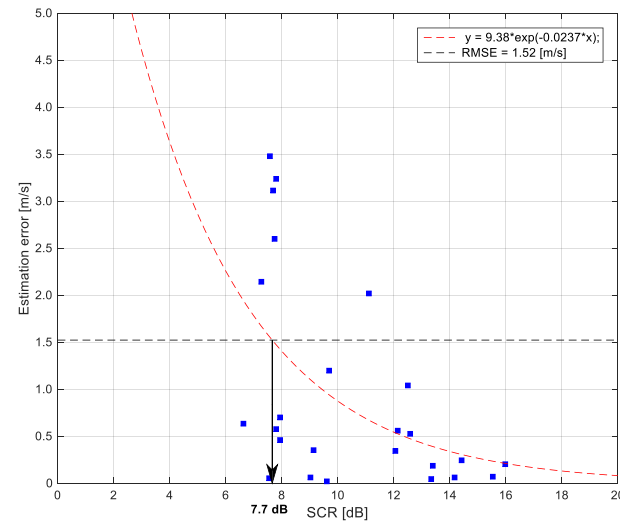

(b)

Figure 3. Determination of optimum conditions for vessel velocity estimation with respect to vessel size and signal-to-clutter ratio (SCR). (a) Size of vessel versus estimation error: The crossing point indicates $19.9 \mathrm{~m}$; thus, a vessel larger than $20 \mathrm{~m}$ is recommended. (b) SCR versus estimation error: The crossing point indicates $7.7 \mathrm{~dB}$; thus, this would be the recommended condition for vessel velocity estimation.

The accuracy of estimation was calculated by using vessels that satisfied the two conditions, i.e., vessel sizes and SCRs larger than $20 \mathrm{~m}$ and $7.7 \mathrm{~dB}$, respectively. The results are displayed in Figure 4 . The squared correlation coefficient $R^{2}$ was 0.89 and 0.97 for the vessel speed and heading angle, respectively. The RMSEs of the speed and heading were $1.09 \mathrm{~m} / \mathrm{s}(2.1 \mathrm{knots})$ and $17.9^{\circ}$ with a slope of 1.05 and 0.94 , respectively. This accuracy would provide useful information for coastal traffic statistics. For the coastal traffic database, the speed, heading, and size of a given vessel are used mainly to determine its type and activities. For instance, most registered crab fishing vessels are heading to the harbor early in the morning according to tidal conditions. If one combines all available information, including the time of day, tidal conditions, permitted fishing season, AIS signals, size of vessel, and its speed and heading, its type and possible activities can be inferred. 


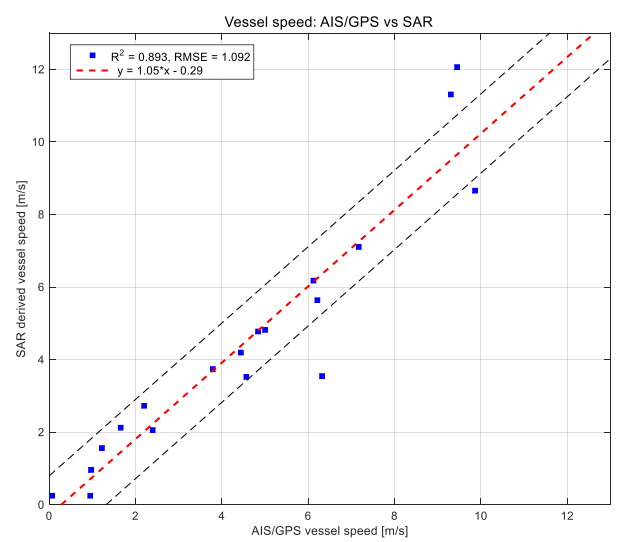

(a)

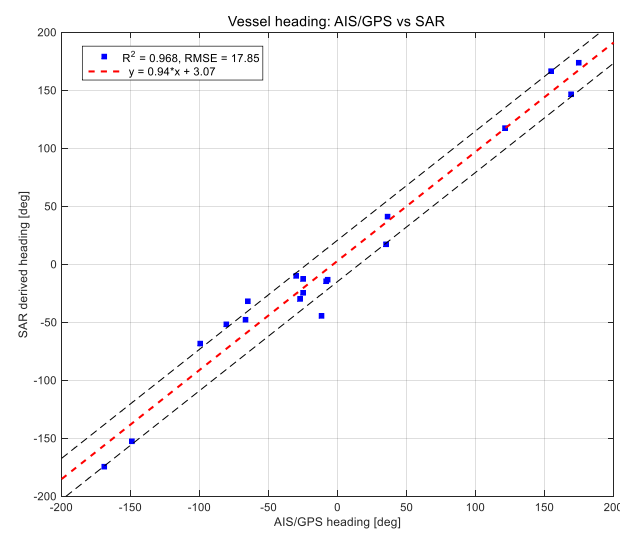

(b)

Figure 4. K5 SAR-derived vessel speed and heading versus AIS/GPS data: (a) Vessel speed and (b) vessel heading. Results were obtained from vessels that satisfied the two conditions of vessel size and SCR larger than $20 \mathrm{~m}$ and $7.7 \mathrm{~dB}$, respectively. The RMSEs of vessel speed and heading angle were $1.09 \mathrm{~m} / \mathrm{s}$ and $17.9^{\circ}$, respectively.

\section{Discussion}

The acceleration of a vessel within the SAR observation period, which is $0.58 \mathrm{~s}$ for the case of the K5 stripmap mode, is usually very small and negligible compared to the speed of the vessel. However, it may not be negligible if there is a significant acceleration/deceleration or a sudden change in heading as the SAR observation occurs. From Equation (8), the comparable radial acceleration to the vessel along-track velocity causing Doppler rate distortion is given by

$$
a_{r}=\frac{2 V_{s}}{R_{0} \sin \theta} v_{a}
$$

Considering a general configuration of the K5 SAR, the value of $\frac{2 V_{S}}{R_{0} \sin \theta}$ is approximately $0.04 \mathrm{sec}^{-1}$ so that the radial acceleration of $a_{r} \simeq 0.04 v_{a}\left(\mathrm{~m} / \mathrm{sec}^{2}\right)$ contribute to the same amount of Doppler rate distortion as that of the along-track velocity $v_{a}(\mathrm{~m} / \mathrm{sec})$. The effects of acceleration were thoroughly discussed by [42] and a sophisticated compensation method was also proposed [43]. However, the method also requires a multi-channel SAR system and uncompressed raw signals. From single-channel SAR systems and SLC data, it is difficult to estimate the acceleration component with a required accuracy. An acceleration analysis method from a single-channel SAR observation was also discussed by [13] for fast-moving vehicles. However, directly applying that method to vessel motion is difficult because the Doppler characteristics of vehicles on land are different from those of vessels at sea. Consequently, the radial acceleration would be one of the main sources of error of the along-track velocity estimation from single-channel spaceborne SAR systems. Figure 5 shows the estimation error with respect to vessel speed and heading with respect to azimuth direction. To examine radar-look direction bias, the vessel heading was transformed to the antenna coordinate direction by which the $0^{\circ}$ matched with the azimuth in Figure 5 . Thus, the $90^{\circ}$ represents the range direction and the $180^{\circ}$ represents the ship approaching the antenna in parallel with the azimuth. The error distribution diagram shows errors larger than $2.1 \mathrm{~m} / \mathrm{s}$ concentrated particularly at speeds higher than $8 \mathrm{~m} / \mathrm{s}$ and approximately $170-240^{\circ}$. There was the largest error on the along-track velocity estimation especially for vessels with high speeds in parallel with the spacecraft flight direction. The main source of error might be the acceleration described in Equations (8) and (16). It implies that the proposed method has a limitation on estimation of the along-track velocity higher than $9 \mathrm{~m} / \mathrm{s}$. It is, however, necessary to do further study with more data to determine a speed limit of estimation, and developing 
a sophisticated method for separately estimating the acceleration from single-channel SAR SLC data must be carried out in the future.

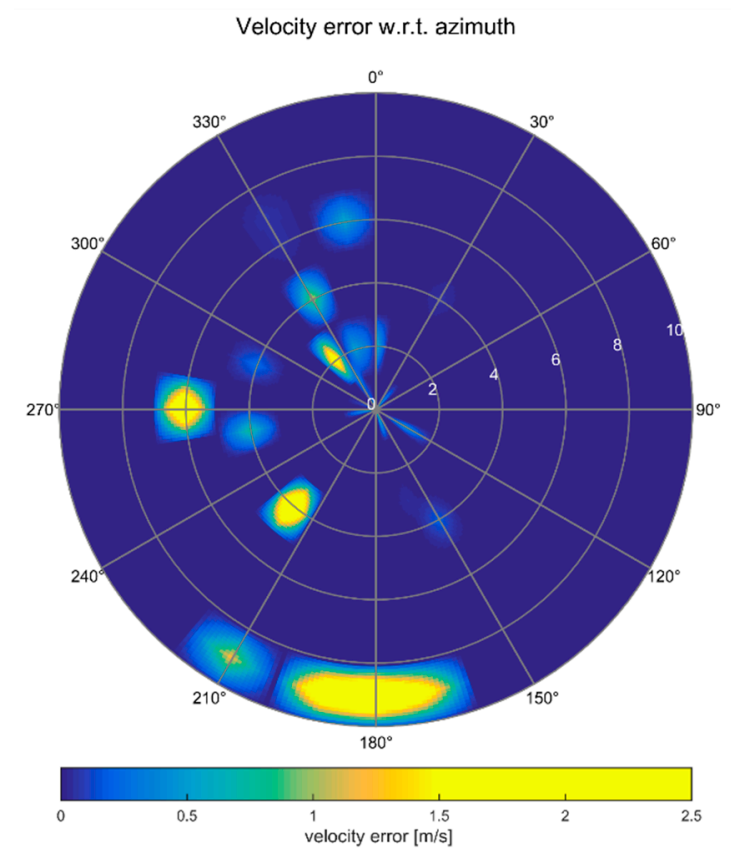

Figure 5. Estimation error of vessel speed according to speed and vessel heading. The heading angles are relative to the spacecraft flight direction. Significant errors occurred at approximately $180^{\circ}$, which is a case of a vessel moving parallel to the flight line in opposite direction to the spacecraft forward motion.

In addition, it is necessary to average a sufficient number of range bins before the Doppler rate estimation because the Doppler frequency rate in Equation (5) is coupled with the range frequency. The zero-range frequency component or DC component can be obtained by averaging the azimuth signals over range bins. For the $\mathrm{K} 5 \mathrm{X}$-band $\mathrm{SAR}$, at least 15 range bins are required for the averaging process.

The potential error sources of the range or radial velocity estimation arise from the additional radial motion of a vessel. Because dominant backscattering occurs in the superstructures of a vessel, additional radial movements of the vessel's high structures and side parts to the antenna look direction would increase the Doppler frequency. When a vessel is imaged by the SAR, the bridge and funnel usually provide a large radar cross section due to corner reflection. The roll motion of a vessel's high structure including the bridge and funnel also produces a higher radial velocity and probably radial acceleration than that of the lower parts. Roll and pitch are common movements for a cruising vessel; high structures such as the bridge and funnel contribute the most to estimating the errors of range velocity. The effects of roll and pitch motion on the SAR-based velocity estimation of ground moving target needs to be quantitatively analyzed in the future. It was, however, reported that a ship roll of $2-3^{\circ}$ would seriously distort Doppler phase and cause blurring of the image of localized scatterers [44,45]. The angular velocity of roll motion is given by [44]

$$
\Omega(t)=\frac{2 \pi A}{T} \cos \left(\frac{2 \pi t}{T}\right)
$$

where $A$ and $T$ are the angular velocity amplitudes of roll and period, respectively. When the roll amplitude and period are $9.6^{\circ}$ and $12 \mathrm{~s}$, respectively, as used by [44], then the angular velocity of roll is given to be $4.85 \mathrm{rad} / \mathrm{s}$ during a $0.5 \mathrm{~s}$ period of SAR integration time. It will produce a perpendicular velocity of $24.28 \mathrm{~m} / \mathrm{s}$ by a scatterer located at $5 \mathrm{~m}$ from the center of mass, and consequently, a radial velocity of $4.05 \mathrm{~m} / \mathrm{s}$ when the rotation axis is normal to the antenna look direction. 
Figure 6 shows an example of an anchored ship. The residual Doppler frequency must be close to zero because the ship remained completely stationary at the moment of the K5 SAR observation. The residual Doppler frequency of this sub-scene was estimated by the $15 \times 15$ window used to produce Figure 6b. As shown in Figure 6b, some high structures produced large Doppler frequencies that originated from the roll and pitch motions of the vessel. Because high structures generally have a large radar cross section, the contribution to the Doppler frequency estimation error is significant. Thus, it is better to estimate the residual Doppler frequency using the radar signals from the freeboard or lower deck of a vessel rather than the signals from the bridge and/or funnel.

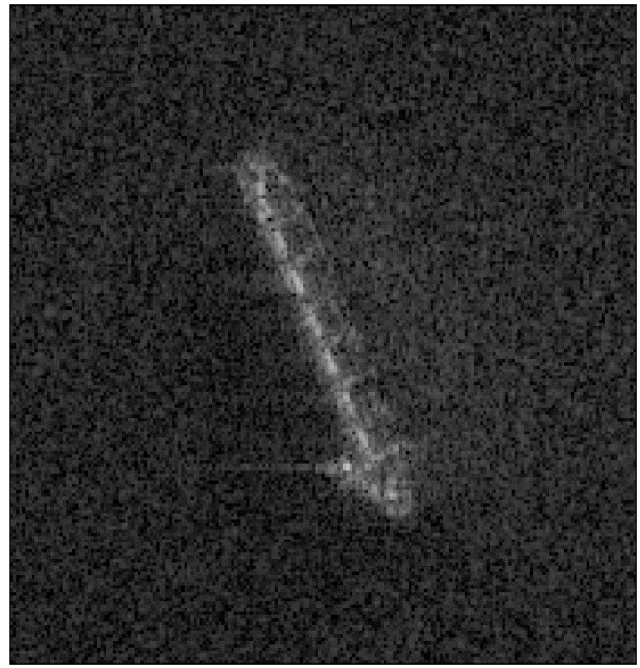

(a)

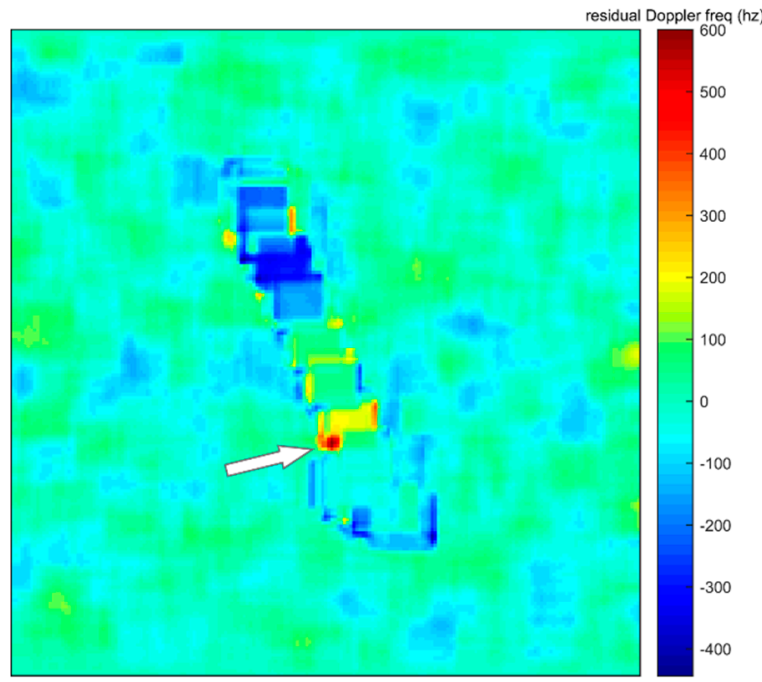

(b)

Figure 6. Example of a residual Doppler frequency caused by roll and pitch motions of vessel: (a) K5 SAR image of a ship anchored at the moment of data acquisition; (b) residual Doppler frequency estimated by a $15 \times 15$ sliding window. This ship was anchored and, thus, stationary at the moment of SAR observation. However, the roll and pitch motions particularly of the vessel high structures contributed to errors of the estimated Doppler frequency; thus, these parts must be excluded in residual Doppler frequency estimation.

It becomes more difficult to precisely measure the vessel speed from SAR observation as the speed decreases. To determine the minimum boundary of K5 SAR-derived vessel speed, stationary vessels commonly observed by AIS and K5 SAR were used for evaluation of measurement accuracy. The uncertainty introduced by the instrumental precision of the AIS measurement is $0.0514 \mathrm{~m} / \mathrm{s}$ [5], but the actual uncertainty of the AIS measured vessel speed would be much larger than this boundary due to unstable vessel motion. Ten vessels with an AIS measured speed less than $0.26 \mathrm{~m} / \mathrm{s}$ (or about 0.5 knots) were used in this study as in Figure 7. A mean value of the 10 AID measured speed was $0.066 \mathrm{~m} / \mathrm{s}$ with a standard deviation of $0.057 \mathrm{~m} / \mathrm{s}$, while the $\mathrm{K} 5 \mathrm{SAR}$-derived speed resulted in a mean speed of $0.64 \mathrm{~m} / \mathrm{s}$ (or about $1.24 \mathrm{knot}$ ) with a standard deviation of $0.34 \mathrm{~m} / \mathrm{s}$. Thus, the method applied to K5 SAR slightly overestimates the speed of stationary vessel, and the radial velocity component contributes more seriously to the errors because of residual Doppler frequency caused by pitch and roll motion. This result implies that the K5 SAR with a proposed method has a limitation of vessel speed less than $0.64 \pm 0.34 \mathrm{~m} / \mathrm{s}$ or roughly 1.9 knots. It is, however, necessary to more precisely determine the minimum boundary of observable speed with a large number of stationary vessels in the future. 


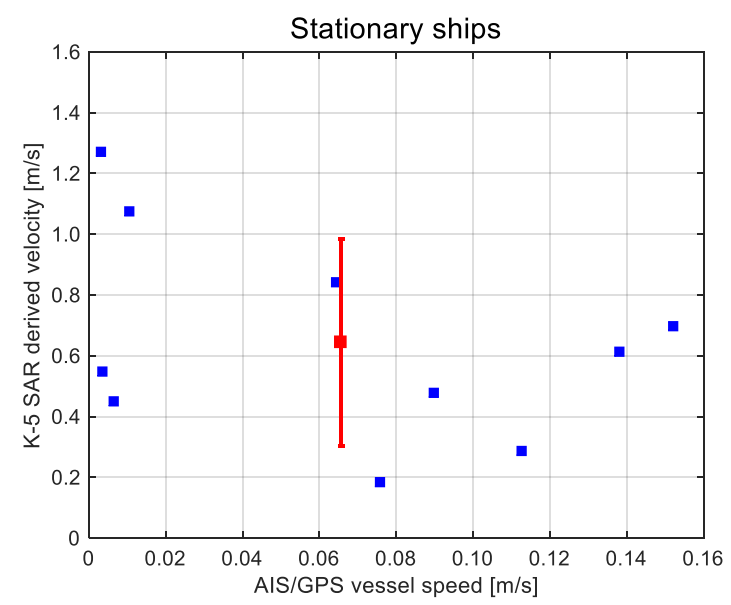

Figure 7. Estimation error bound of vessel speed for stationary vessels. The mean speed of K5 SAR-derived stationary vessel was $0.64 \mathrm{~m} / \mathrm{s}$ with a standard deviation of $0.340 \mathrm{~m} / \mathrm{s}$ while the mean AIS measured speed was $0.066 \mathrm{~m} / \mathrm{s}$ with a standard deviation of $0.057 \mathrm{~m} / \mathrm{s}$. The error bar indicates the mean and standard deviation of the K5 SAR measurement.

\section{Conclusions}

The velocity and heading of vessels are important data for monitoring and controlling coastal areas. The AIS alone is not enough for collecting information on vessels and accumulating a coastal traffic database, but spaceborne SAR systems are very effective and efficient supplementary tools for this purpose. Estimating the vessel velocity two-dimensionally from a K5 X-band SAR system was successful. Both the ground range (or radial) and azimuth (or along-track) velocities were estimated from the SAR SLC data, from which the speed and heading were consequentially obtained. Through two field campaigns carried out in 2017 and 2018, the measurement accuracy was evaluated. The minimum size of vessel and SCR were determined to be $20 \mathrm{~m}$ and $7.7 \mathrm{~dB}$, respectively. From a total of 19 vessels that satisfied the minimum size of vessel and SCR, the squared correlation coefficient $R^{2}$ were 0.89 and 0.97 for the vessel speed and heading angle, respectively, and the RMSEs of the speed and heading were $1.09 \mathrm{~m} / \mathrm{s}(2.1 \mathrm{knots})$ and $17.9^{\circ}$, respectively. In fact, the K5 SAR has a capability for observing a selected coastal region once a day by utilizing various modes, and therefore, it is possible to accumulate a large amount of vessel information from the coastal sea eventually for building a coastal traffic model. The method and results will be applied to a selected coastal sea in the future and the spaceborne SAR application capability for building a coastal traffic model will be demonstrated.

Although the method applied to $\mathrm{K} 5$ was successful for retrieving a two-dimensional ship velocity, further research is required for detecting and monitoring small vessels with slow speed. Radial acceleration is the main source of error on the along-track velocity estimation. Acceleration estimation from single-channel SAR SLC data is still neither efficient nor effective. High-speed vessels moving parallel to the antenna flight line are still problematic, and this is a future task to be resolved. Concerning the range velocity estimation, the roll and pitch motions of a vessel are the main contributors to these errors. Particularly, the bridge and funnel produce an additional range velocity component while they play an important role as backscatterers. In the near future, reducing the effects of distorted signals caused by the roll and pitch motions particularly from such high structures on the range velocity estimation is necessary.

Author Contributions: Conceptualization, J.-S.W.; methodology, J.-S.W. and M.B.; validation, D.K. and S.-W.K.; formal analysis, M.B., J.-S.W. and S.-W.K.; investigation, M.B., D.K., S.-W.K. and J.-S.W.; data curation, M.B. and D.K.; writing — original draft preparation, J.-S.W.; writing—review and editing, J.-S.W.; visualization, S.-W.K. and J.-S.W.; project administration, J.-S.W. and S.-W.K.; funding acquisition, J.-S.W. and S.-W.K.

Funding: This research was financially supported by the Korea Institute of Marine Science \& Technology Promotion funded by the Ministry of Ocean and Fisheries for the "Base research for building a wide integrated surveillance system of marine territory" project. 
Acknowledgments: The authors sincerely appreciate the Korea Institute of Ocean Science \& Technology (KIOST) for providing the ship motion data and in-situ data as part of the field campaigns carried out in 2017 and 2018. The authors would like to thank the Korea Aerospace Research Institute (KARI) for acquisition and provision of KOMSAT-5 SAR data.

Conflicts of Interest: The authors declare no conflict of interest.

\section{References}

1. Brusch, S.; Lehner, S.; Fritz, T.; Soccorsi, M.; Soloviev, A.; van Schie, B. Ship Surveillance with TerraSAR-X. IEEE Trans. Geosci. Remote Sens. 2011, 49, 1092-1103. [CrossRef]

2. Renga, A.; Moccia, A. Use of Doppler Parameters for Ship Velocity Computation in SAR Images. IEEE Trans. Geosci. Remote Sens. 2016, 54, 3995-4011. [CrossRef]

3. Panico, A.; Graziano, M.D.; Renga, A. SAR-Based Vessel Velocity Estimation from Partially Imaged Kelvin Pattern. IEEE Geosci. Remote Sens. Lett. 2017, 14, 2067-2071. [CrossRef]

4. Graziano, M.D.; D’Errico, M.; Rufino, G. Wake Component Detection in X-Band SAR Images for Ship Heading and Velocity Estimation. Remote Sens. 2016, 8, 498. [CrossRef]

5. Ao, D.Y.; Datcu, M.; Schwarz, G.; Hu, C. Moving Ship Velocity Estimation Using TanDEM-X Data Based on Subaperture Decomposition. IEEE Geosci. Remote Sens. Lett. 2018, 15, 1560-1564. [CrossRef]

6. Raney, R.K. Synthetic Aperture Imaging Radar and Moving Targets. IEEE Trans. Aerosp. Electron. Syst. 1971, 7, 499-505. [CrossRef]

7. Kirscht, M. Detection and imaging of arbitrarily moving targets with single-channel SAR. IEE Proc. Radar Sonar Navig. 2003, 150, 7-11. [CrossRef]

8. Won, J.S. Doppler Frequency Estimation of Point Targets in the Single-Channel SAR Image by Linear Least Squares. Remote Sens. 2018, 10, 1160. [CrossRef]

9. Ouchi, K. On the multilook images of moving targets by synthetic aperture radars. IEEE Trans. Antennas Propag. 1985, 33, 823-827. [CrossRef]

10. Myronenko, A.; Song, X.B. Point Set Registration: Coherent Point Drift. IEEE Trans. Pattern Anal. Mach. Intell. 2010, 32, 2262-2275. [CrossRef]

11. Park, J.W.; Kim, J.H.; Won, J.S. Fast and Efficient Correction of Ground Moving Targets in a Synthetic Aperture Radar, Single-Look Complex Image. Remote Sens. 2017, 9, 926. [CrossRef]

12. Madsen, S.N. Estimating the Doppler Centroid of Sar Data. IEEE Trans. Aerosp. Electron. Syst. 1989, 25, 134-140. [CrossRef]

13. Park, J.W.; Won, J.S. An Efficient Method of Doppler Parameter Estimation in the Time-Frequency Domain for a Moving Object from TerraSAR-X Data. IEEE Trans. Geosci. Remote Sens. 2011, 49, 4771-4787. [CrossRef]

14. Li, F.K.; Held, D.N.; Curlander, J.C.; Wu, C. Doppler Parameter-Estimation for Spaceborne Synthetic-Aperture Radars. IEEE Trans. Geosci. Remote Sens. 1985, 23, 47-56. [CrossRef]

15. Bamler, R. Doppler Frequency Estimation and the Cramer-Rao Bound. IEEE Trans. Geosci. Remote Sens. 1991, 29, 385-390. [CrossRef]

16. Wong, F.; Cumming, I.G. A combined SAR Doppler centroid estimation scheme based upon signal phase. IEEE Trans. Geosci. Remote Sens. 1996, 34, 696-707. [CrossRef]

17. Li, W.C.; Yang, J.Y.; Huang, Y.L. Improved Doppler parameter estimation of squint SAR based on slope detection. Int. J. Remote Sens. 2014, 35, 1417-1431. [CrossRef]

18. Marques, P.A.C.; Dias, J.M.B. Velocity estimation of fast moving targets using a single SAR sensor. IEEE Trans. Aerosp. Electron. Syst. 2005, 41, 75-89. [CrossRef]

19. Chen, C.C.; Andrews, H.C. Target-Motion-Induced Radar Imaging. IEEE Trans. Aerosp. Electron. Syst. 1980, 16, 2-14. [CrossRef]

20. Barbarossa, S. Detection and Imaging of Moving-Objects with Synthetic Aperture Radar. Part 1: Optimal Detection and Parameter-Estimation Theory. IEE Proc. F Radar Signal Process. 1992, 139, 79-88. [CrossRef]

21. Barbarossa, S.; Farina, A. Detection and Imaging of Moving-Objects with Synthetic Aperture Radar. Prat 2: Joint Time Frequency-Analysis by Wigner-Ville Distribution. IEE Proc. F Radar Signal Process. 1992, 139, 89-97. [CrossRef]

22. Tunaley, J.K.E. The estimation of ship velocity from SAR imagery. In Proceedings of the IGARSS 2003, Toulous, France, 21-25 July 2003; pp. 91-93. 
23. Graziano, M.D.; Grasso, M.; D’Errico, M. Performance Analysis of Ship Wake Detection on Sentinel-1 SAR Images. Remote Sens. 2017, 9, 1107. [CrossRef]

24. Meyer, F.; Hinz, S.; Laika, A.; Suchandt, S.; Bamler, R. Performance analysis of space-borne SAR vehicle detection and velocity estimation. In Proceedings of the ISPRS Commission VII Symposium, Born, Germany, 20-22 October 2006.

25. Abatzoglou, T.J. Fast Maximum-Likelihood Joint Estimation of Frequency and Frequency Rate. IEEE Trans. Aerosp. Electron. Syst. 1986, 22, 708-715. [CrossRef]

26. Peleg, S.; Porat, B. Linear Fm Signal Parameter-Estimation from Discrete-Time Observations. IEEE Trans. Aerosp. Electron. Syst. 1991, 27, 607-616. [CrossRef]

27. O'Shea, P. A new technique for instantaneous frequency rate estimation. IEEE Signal Proc. Lett. 2002, 9, 251-252. [CrossRef]

28. O'Shea, P. A fast algorithm for estimating the parameters of a quadratic FM signal. IEEE Trans. Signal Process. 2004, 52, 385-393. [CrossRef]

29. O'Shea, P. On Refining Polynomial Phase Signal Parameter Estimates. IEEE Trans. Aerosp. Electron. Syst. 2010, 46, 978-987. [CrossRef]

30. Barbarossa, S.; Farina, A. Space-Time-Frequency Processing of Synthetic-Aperture Radar Signals. IEEE Trans. Aerosp. Electron. Syst. 1994, 30, 341-358. [CrossRef]

31. Kersten, P.R.; Jansen, R.W.; Luc, K.; Ainsworth, T.L. Motion analysis in SAR images of unfocused objects using time-frequency methods. IEEE Geosci. Remote Sens. Lett. 2007, 4, 527-531. [CrossRef]

32. Kersten, P.R.; Toporkov, J.V.; Ainsworth, T.L.; Sletten, M.A.; Jansen, R.W. Estimating Surface Water Speeds with a Single-Phase Center SAR Versus an Along-Track Interferometric SAR. IEEE Trans. Geosci. Remote Sens. 2010, 48, 3638-3646. [CrossRef]

33. Almeida, L.B. The Fractional Fourier-Transform and Time-Frequency Representations. IEEE Trans. Signal Process. 1994, 42, 3084-3091. [CrossRef]

34. Clemente, C.; Soraghan, J.J. Range Doppler and chirp scaling processing of synthetic aperture radar data using the fractional Fourier transform. IET Signal Process. 2012, 6, 503-510. [CrossRef]

35. Yetik, I.S.; Nehorai, A. Beamforming using the fractional Fourier transform. IEEE Trans. Signal Process. 2003, 51, 1663-1668. [CrossRef]

36. Chiu, S. Application of fractional Fourier transform to moving target indication via along-track interferometry. Eur. J. Appl. Signal Process. 2005, 2005, 3293-3303. [CrossRef]

37. Elgamel, S.A.; Soraghan, J. Enhanced monopulse tracking radar using optimum fractional Fourier transform. IET Radar Sonar Navig. 2011, 5, 74-82. [CrossRef]

38. Xi, L.; Guosui, L.; Ni, J.L. Autofocusing of ISAR images based on entropy minimization. IEEE Trans. Aerosp. Electron. Syst. 1999, 35, 1240-1252. [CrossRef]

39. Zeng, T.; Wang, R.; Li, F. SAR Image Autofocus Utilizing Minimum-Entropy Criterion. IEEE Geosci. Remote Sens. Lett. 2013, 10, 1552-1556. [CrossRef]

40. Kragh, T.J. Monotonic iterative algorithm for minimum-entropy autofocus. In Proceedings of the 14th Adaptive Sensor Array Processing (ASAP) Workshop, Lexington, MA, USA, 6-7 June 2006.

41. Raney, R.K. Doppler Properties of Radars in Circular Orbits. Int. J. Remote Sens. 1986, 7, 1153-1162. [CrossRef]

42. Sharma, J.J.; Gierull, C.H.; Collins, M.J. The influence of target acceleration on velocity estimation in dual-channel SAR-GMTI. IEEE Trans. Geosci. Remote Sens. 2006, 44, 134-147. [CrossRef]

43. Sharma, J.J.; Gierull, C.H.; Collins, M.J. Compensating the effects of target acceleration in dual-channel SAR-GMTI. IEE Proc. Radar Sonar Navig. 2006, 153, 53-62. [CrossRef]

44. Berizzi, F.; Diani, M. Target angular motion effects on ISAR imaging. IEE Proc. Radar Sonar Navig. 1997, 144, 87-95. [CrossRef]

45. Given, J.A.; Schmidt, W.R. Generalized ISAR_Part I: An optimal method for imaging large naval vessels. IEEE Trans. Image Process. 2005, 14, 1783-1791. [CrossRef] [PubMed]

(C) 2019 by the authors. Licensee MDPI, Basel, Switzerland. This article is an open access article distributed under the terms and conditions of the Creative Commons Attribution (CC BY) license (http://creativecommons.org/licenses/by/4.0/). 